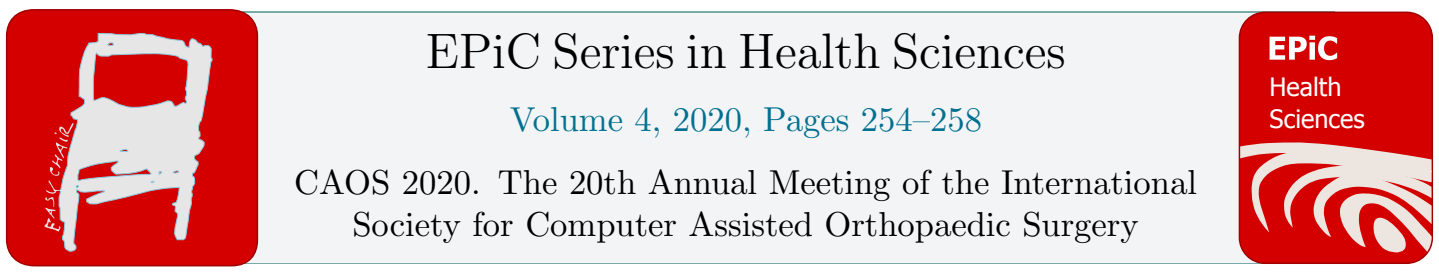

\title{
Post-Operative Virtual Range of Motion in Robotic-Assisted THA Patients to Determine Pre-Operative Simulation Needs
}

\author{
Matthew Thompson ${ }^{1 *}$, Roopa Guttal ${ }^{1}$, Shon Darcy ${ }^{1}$, Akshay Alaghatta $^{1}$, \\ Andrea Marcovigi ${ }^{2}$, Fabio Catani ${ }^{2 * *}$ \\ ${ }^{1}$ Stryker, Fort Lauderdale, FL, USA \\ ${ }^{2}$ Department of Orthopaedic Surgery, University of Modena and Reggio-Emilia, Italy \\ *matt.thompson@stryker.om, **FCatani59@gmail.com
}

\begin{abstract}
For preoperative simulations of hip range of motion to be useful in predicting complications after total hip arthroplasty (THA), the factors that could affect post-operative function must be considered including, but not limited to, bony impingement, pelvic position, and implanted vs. planned differences. This study retrospectively simulates ranges of motion to prosthetic and bony impingement of THA patients with known planned and implanted component positions and pelvic tilt to determine the factors and needs to accurately simulate range of motion preoperatively.

Twenty-two (22) anterolateral, cementless total hip arthroplasties were performed using robotic-arm assisted technology which allowed capture of the implanted stem version and position in addition to robotic-assisted cup placement to plan. With the known implanted positions and preoperative 3-dimentional (3D) bone models, six (6) hip maneuvers were virtually simulated in custom software. Correlations were evaluated between planned and implanted component positions, pelvic tilt, ranges of motion, and patient-reported outcomes.

Average ranges of motion to impingement were similar to those of previous simulation and navigation studies. Supine tilt varied from $-10^{\circ}$ (posterior) to $15^{\circ}$ (anterior) with an average of $3.4 \pm 6.6^{\circ}$. Very little correlation was seen between native or planned stem version and implanted stem version. Correlations were seen between some maneuvers such as internal rotation (IR) at 90 degrees flexion (F) (IR@90F) and combined component version and pelvic tilt. Bony impingement occurred during IR@90F in 9 of the 22 cases. Pelvic tilt assessment, bony impingement detection, better prediction of implanted component position or the ability to execute a plan, such as robotically, would all provide a more accurate pre-operative simulation of the post-operative patient's function.
\end{abstract}




\section{Introduction}

Simulations of range of motion of the hip to impingement have been extensively reported in the literature and can be useful in predicting complications after total hip arthroplasty (THA) such as dislocation. ${ }^{1-}$

${ }^{4}$ However, many of these studies focus only on prosthetic impingement ${ }^{1}$ or cadaveric bone models, ${ }^{3,4}$ do not evaluate the pelvic position of the patient, and/or ignore the actual errors in component implantation needed for accurate pre-operative simulation.

This study retrospectively determines possible errors in component placement relative to the plan and simulates ranges of motion to both prosthetic and bony impingement of THA patients with known component and pelvic positions to determine the factors and needs to accurately simulate range of motion pre-operatively. Patient-reported outcomes were also obtained to determine if any correlations exist with their calculated ranges of motions.

\section{Methods}

Twenty-two (22) total hip arthroplasties were performed by the senior author using robotic-arm assisted technology. All 22 hips were performed through an anterolateral approach and utilized an enhanced femoral workflow which allowed capture of the implanted stem version (prepared and implanted through manual means) in addition to robotic-assisted cup placement to plan. Thirteen (13) of the hips were implanted with a tapered-wedge stem and nine (9) were implanted with an anatomic fit-and-fill stem. Nineteen (19) were implanted with a neutral liner and 3 were implanted with a dualmobility liner.

Post-operatively, data was extracted from the robotic patient session files in order to extract case information such as the pre-operative 3-dimentionsl (3D) bone models and planned and implanted stem and cup types, sizes, positions, and orientations. Pre and post-operative patient reported outcomes were also obtained (Western Ontario and McMaster Universities Osteoarthritis Index (WOMAC) and Harris Hip Score).

Patients' robotic files were then imported into custom virtual range of motion software capable of realigning the planned implant models to their intra-operatively captured positions. The software can then simulate range of motion routines that detect bony and prosthetic impingement by controlling flexion/extension, internal/external rotations, and abduction/adduction of the hip in one degree increments. Six (6) maneuvers were simulated until impingements were observed: maximum flexion, extension, internal rotation (IR) at 0 degrees of flexion (F) (IR@0F), external rotation (ER) at 0 degrees of flexion (ER@0F), internal rotation at 90 degrees of flexion (IR@90F), and external rotation at 20 degrees of extension (E) (ER@20E). Osteophyte impingement was ignored as it was assumed these would have been removed intra-operatively.

Average ranges of motion to impingement and the incidence of bony impingement prior to prosthetic impingement and vice versa were calculated. Correlations were evaluated between native, planned, and implanted component positions and between ranges of motion and component positions, pelvic tilt, and patient-reported outcomes.

\section{Results}

Average implanted radiographic cup inclination and version were $40 \pm 1^{\circ}$ and $21 \pm 3^{\circ}$, respectively. Average implanted stem version was $12 \pm 9^{\circ}$ (range: $-4^{\circ}$ to $31^{\circ}$ ) leading to an average combined version of $33 \pm 6^{\circ}$. Average supine pelvic tilt was calculated as $3.4 \pm 6.6^{\circ}$ anteriorly with a range from $10^{\circ}$ 
posterior tilt to $15^{\circ}$ anterior tilt. Average ranges of motions to initial impingement were $114 \pm 8^{\circ}$ of flexion, $49 \pm 7^{\circ}$ of extension, $48 \pm 7^{\circ}$ of ER@0F, 32 $\pm 11^{\circ}$ of IR@90F, and 27 $\pm 8^{\circ}$ of ER@20E. Impingement in IR@0F occurred at greater than $90^{\circ}$ for all hips.

Very little correlation was seen between native and implanted stem version $(\mathrm{r} 2=0.16)$ and between planned and implanted stem version $(\mathrm{r} 2=0.04)$. In the IR@90F maneuver, bony impingement (typically the anterior neck or greater trochanter against the inferior iliac spine) was observed prior to prosthetic impingement in 9 of the 22 cases (Average IR@90F to bony impingement $=37 \pm 14^{\circ}$; IR@90F to liner impingement $=36 \pm 8^{\circ}$ ). In the ER@20E maneuver, bony impingement (typically the lesser trochanter against the anterior ischium, Figure 1) was observed prior to prosthetic impingement in only 3 of the 22 cases (Average ER@20E to bony impingement $=42 \pm 15^{\circ}$; ER@20E to liner impingement $=29 \pm 7^{\circ}$ ).

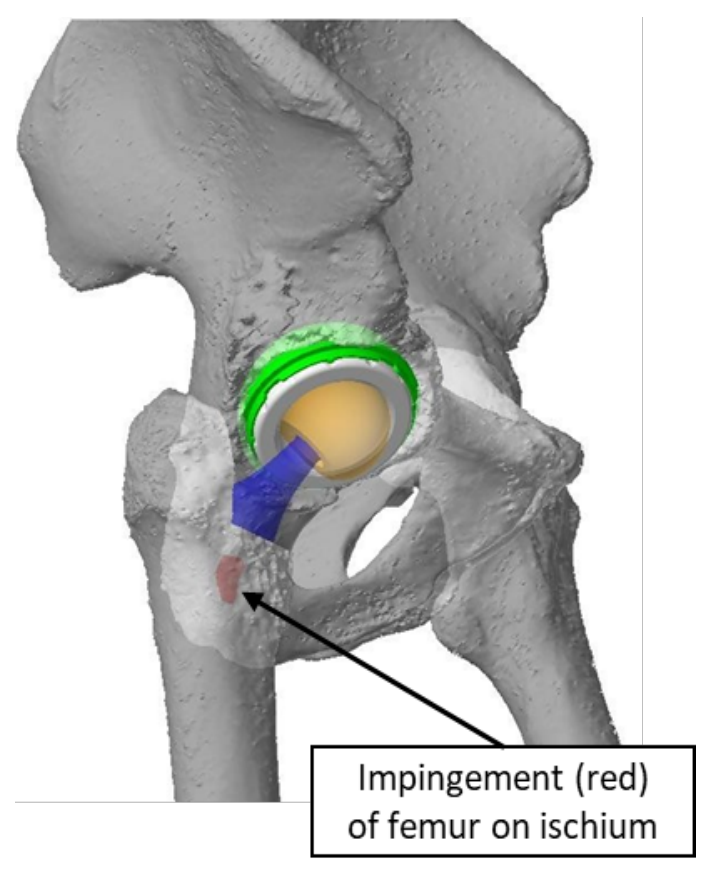

Figure 1. Example of detected bony impingement on the ischium (in red) during external rotation in $20^{\circ}$ of extension.

Internal rotation at 90 degrees of flexion (IR@90F) was found to inversely correlate with increasing anterior pelvic tilt $(\mathrm{r} 2=0.25)$ and positively with combined anteversion $(\mathrm{r} 2=0.57$, Figure 2$)$. Even when ignoring prosthetic impingement, IR@90F to bony impingement correlated well with stem version $(\mathrm{r} 2=0.55)$. External rotation at 20 degrees of extension $(\mathrm{ER} @ 20 \mathrm{E})$ inversely correlated with combined component anteversion ( $\mathrm{r} 2=0.42$, Figure 2$)$. 

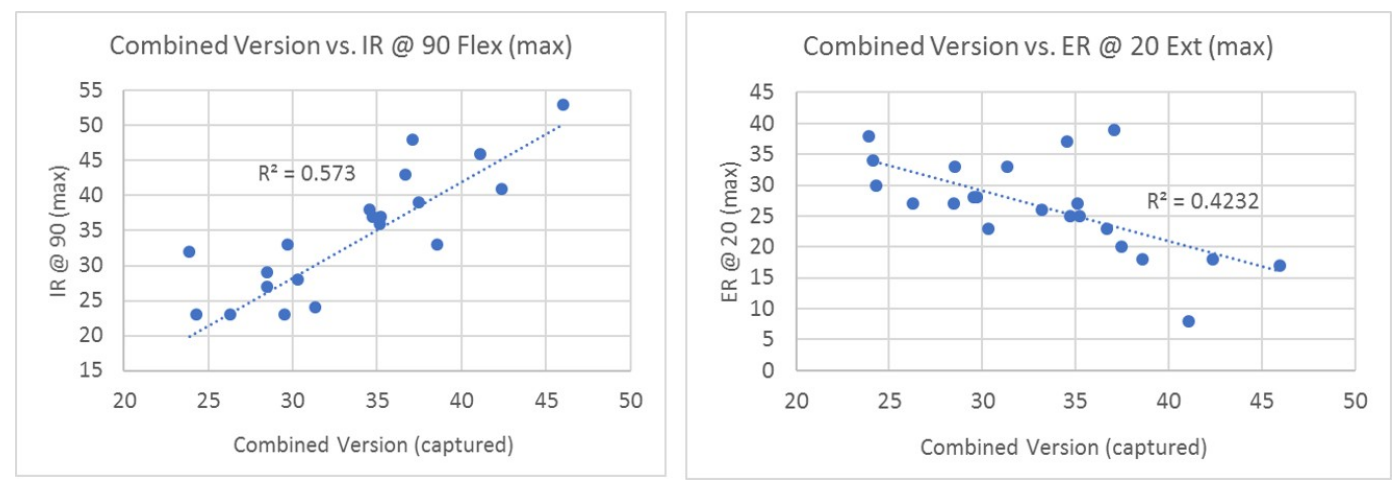

Figure 2. Even when considering cases of bony impingement, increasing combined component anteversion correlates well with increasing internal rotation at 90 degrees of flexion and decreasing external rotation at 20 degrees of extension.

Weak correlations were seen between IR@90F and pre and post-op WOMAC differences $(\mathrm{r} 2=0.04)$ and between hip length discrepancy (HLD) and post-op WOMAC scores ( $\mathrm{r} 2=0.04)$, but trendlines were in the expected direction (i.e., increasing IR@90F with more negative WOMAC differences and increasing HLD with increasing post-op WOMAC score).

\section{Discussion}

The average range of motion values determined in this study correlate well with the common THA maneuvers simulated by Klingenstein et al. and Miki et al. (flexion, extension, and ER@0)., Several other results of this study were as one would expect or as previously reported (e.g., increasing range of motion in flexion maneuvers and decreasing range of motion in extension maneuvers with increasing combined anteversion). ${ }^{6,7}$ However, some less obvious results were also observed. For example, decreasing IR@90F with increasing anterior pelvic tilt, a lack of correlation between planned stem or native version and final stem version, and increasing IR@90F to bony impingement with increasing stem version were all observed. Bony impingement was also shown to frequently occur prior to component impingement in flexion maneuvers as also reported by Kessler et al. ${ }^{8}$

An obvious limitation of this study and many virtual range-of-motion studies of the hip is the lack of soft-tissue simulation. While the contribution of soft-tissue tension and other soft-tissue constraints prior to impingement can vary greatly by patient and maneuver, ${ }^{2,9}$ Turley et al. stated that "in CT simulations, range of motion restriction slightly overestimates the required range of motion due to the absence of soft tissue by $5^{\circ} .{ }^{\prime 2}$

Given the small sample size of this study and the multitude of factors that define a successful hip replacement, it was not surprising that very little correlation was seen between range of motion and patient-reported outcomes (PROMs). Future studies would benefit from a larger sample size with greater focus on the functional portions of patient-reported outcomes to determine if correlations with PROMs exist. 


\section{Conclusion}

This study post-operatively assessed implanted total hip components in total hip patients to determine factors that contribute to their simulated ranges of motion. For virtual range of motion to be a useful pre-operative tool, these factors must be accurately simulated with a means to execute the preoperative plan. Pelvic tilt assessment, bony impingement detection, better prediction of implanted component position or the ability to execute a plan, such as robotically, would all provide a more accurate pre-operative simulation of the post-operative patient's range of motion, hip function, and satisfaction.

\section{References}

1. McCarthy TF, Alipit V, Nevelos J, Elmallah RK, Mont MA. Acetabular Cup Anteversion and Inclination in Hip Range of Motion to Impingement. J Arthroplasty. 2016 Sep;31(9 Suppl):2648

2. Turley GA, Williams MA, Wellings RM, Griffin DR. Evaluation of range of motion restriction within the hip joint. Med Biol Eng Comput. 2013;51(4):467-477

3. Klingenstein GG, Yeager AM, Lipman JD, Westrich GH. Computerized range of motion analysis following dual mobility total hip arthroplasty, traditional total hip arthroplasty, and hip resurfacing. J Arthroplasty. 2013 Aug;28(7):1173-6

4. Klingenstein GG, Yeager AM, Lipman JD, Westrich GH. Increased range of motion to impingement with large head total hip arthroplasty: point of diminishing returns. Hip Int. 2012 May- Jun;22(3):261-5

5. Miki H, Yamanashi W, Nishii T, Sato Y, Yoshikawa H, Sugano N. Anatomic hip range of motion after implantation during total hip arthroplasty as measured by a navigation system. J Arthroplasty. 2007 Oct;22(7):946-52

6. Ohmori T, Kabata T, Kajino Y, Inoue D, Taga T, Yamamoto T, Takagi T, Yoshitani J, Ueno T, Ueoka $\mathrm{K}$, Tsuchiya $\mathrm{H}$. The optimal combined anteversion pattern to achieve a favorable impingement-free angle in total hip arthroplasty. J Orthop Sci. 2019 May;24(3):474-481

7. Dorr LD, Malik A, Dastane M, Wan Z. Combined anteversion technique for total hip arthroplasty. Clin Orthop Relat Res. 2009;467(1):119-127

8. Kessler O, Patil S, Wirth S, Mayr E, Colwell CW Jr, D'Lima DD. Bony impingement affects range of motion after total hip arthroplasty: A subject-specific approach. J Orthop Res. 2008 Apr;26(4):443-52

9. Han S, Owens V, Patel RV, Ismaily SK, Harrington MA, Incavo SJ, Noble PC. The Continuum of Hip Range of Motion: From Soft-Tissue Restriction to Bony Impingement. J Orthop Res. 2020 Jan 22. 